\title{
Estresse ocupacional: fatores de risco para os profissionais da medicina e da enfermagem
}

\author{
Occupational stress: risk factors for medicine and nursing professionals
}

Estrés ocupacional: factores de riesgo para los profesionales de medicina y enfermería

Lorena de Almeida Ludovino $^{1 *}$, João Pedro Reis de Almeida ${ }^{1}$, Fernanda Luiza Andrade de Azevedo .

\section{RESUMO}

Objetivo: Identificar os principais agentes estressores responsáveis pelo transtorno do estresse laboral agudo e crônico em profissionais médicos e enfermeiros, bem como analisar e descrever o impacto da COVID 19 na saúde mental. Revisão bibliográfica: $O$ estresse é uma reação normal de adaptação do organismo frente aos diversos estímulos ambientais. No entanto, se persistente por mais de 4 semanas, o estresse torna-se crônico e patológico ocasionando desde de Hipertensão Arterial, a Ansiedade e Depressão. O transtorno experimentado no ambiente laboral é definido como ocupacional. No presente estudo, os médicos e os enfermeiros são detentores de injúrias físicas e mentais como ansiedade, cansaço e ideação suicida em taxas superiores a maioria da população em geral, associado ainda aos impactos negativos da COVID, uma vez que lidar com o desconhecido e ao mesmo tempo tentar manter o equilíbrio para cuidar do outro, tornou-se o maior dos desafios. Considerações finais: Logo, os profissionais médicos e enfermeiros figuram entre as classes mais suscetíveis ao desenvolvimento do estresse laboral. Assim sendo, mudanças que visam o cuidado com a saúde física e mental são necessárias, a fim de garantir não somente o bem estar dos profissionais, mas também a manutenção na qualidade da assistência.

Palavras-chave: Saúde mental, Estresse ocupacional, Medicina, Enfermagem.

\begin{abstract}
Objective: To identify the main stressors agents responsible for acute and chronic work stress disorder in medical professionals and nurses, as well as to analyze and describe the impact of COVID-19 on mental health. Bibliographic review: Stress is a normal adaptation reaction of the organism to various environmental stimuli. However, if persistent for more than 4 weeks, stress becomes chronic and pathological, causing from Hypertension to Anxiety and Depression. The disorder experienced in the work environment is defined as occupational. In this study, doctors and nurses have physical and mental injuries such as anxiety, fatigue and suicidal ideation at higher rates than most of the general population, also associated with the negative impacts of COVID, since dealing with the unknown and at the same time trying to maintain balance to care for the other, has become the biggest challenge. Final considerations: Thus, medical and nursing professionals are among the classes most susceptible to the development of work stress. Therefore, changes aimed at caring for physical and mental health are necessary, in order to ensure not only the well-being of professionals, but also the maintenance of the quality of care.
\end{abstract}

Keywords: Mental health, Occupational stress, Medicine, Nursing.

\section{RESUMEN}

Objetivo: Identificar los principales factores estresantes responsables por el trastorno del estrés ocupacional agudo y crónico en profesionales médicos y enfermeros, así como analizar y describir el impacto del COVID19 en la salud mental. Revisión bibliográfica: El estrés es una reacción de adaptación normal del cuerpo a diversos estímulos ambientales. Sin embargo, si persiste durante más de 4 semanas, el estrés se vuelve

\footnotetext{
${ }^{1}$ Faculdades Santo Agostinho (FASAI), Itabuna - BA. *E-mail: Iorenaludovino@hotmail.com
} 
crónico y patológico provocando desde hipertensión hasta ansiedad y depresión. El trastorno experimentado en el entorno laboral se define como ocupacional. En este estudio, médicos y enfermeras presentan lesiones físicas y mentales como ansiedad, cansancio e ideación suicida en tasas superiores a la mayoría de la población en general, asociadas también a los impactos negativos del COVID, ya que lidiar con lo desconocido y al mismo tiempo tratar de mantener el equilibrio para cuidar al otro, se ha convertido en el mayor desafío. Consideraciones finales: Por lo tanto, los profesionales médicos y enfermeros se encuentran entre las clases más susceptibles al desarrollo de estrés laboral. Así, los cambios dirigidos al cuidado de la salud física y mental son necesarios para asegurar no solo el bienestar de los profesionales, sino también el mantenimiento de la calidad asistencial.

Palabras clave: Salud mental, Estrés ocupacional, Medicina, Enfermería.

\section{INTRODUÇÃO}

A sociedade pós revolução industrial passou por transformações sociais e tecnológicas, as quais foram de suma importância para a reorganização do trabalho, aumento da produtividade nas empresas/serviços, além de impactar de maneira abrangente a vida do trabalhador (SILVA GN, 2019). Esse novo cenário, altamente competitivo, responde pelo desgaste físico e mental, uma vez que o ambiente laboral tornou-se adoecedor (PRADO CEP, 2016).

Hans Selye, em sua teoria biológica descreveu o estresse nas etapas de Alerta, Resistência e Exaustão. Entre 1970 e 1980 seus estudos foram aprofundados por Lazarus, Lanier e Folkman e correlacionados com os aspectos cognitivos e comportamentais, vide a sua relação com o meio. Em 2000, a pesquisadora brasileira Marilda Lipp ampliou esse modelo para quadrifásico: Alerta, Resistência, Quase Exaustão e Exaustão (BANDEIRA MAD, 2017).

Define-se estresse como uma reação do corpo frente aos estímulos que provocam alterações físicas e psicológicas. Em níveis normais é essencial para a sobrevivência do ser humano, no entanto, se experimentado de forma intensa e recorrente, torna-se patológico sendo classificado como transtorno do estresse agudo ou crônico. Sob essa égide, se essa vivência for no ambiente laboral, o estresse é entendido como ocupacional, principal agente danoso à saúde do trabalhador (SADIR MA, et al., 2010).

Os profissionais da medicina e da enfermagem, sobretudo aqueles que trabalham em ambientes hospitalares estão entre os mais vulneráveis ao desenvolvimento do transtorno do estresse, uma vez que lidam diuturnamente com a dor do outro, com o sofrimento e a morte. Aliado a isso, os baixos salários, a jornada exaustiva, a falta de insumos e recursos humanos (RIBEIRO RP, et al., 2018) corroboram para aumentar o risco de acidentes ocupacionais em trabalhadores com mais de 40 horas semanais (HONORATO CMA e AZEVEDO FC, et al., 2019).

O estresse persistente pode desencadear patologias físicas e mentais como hipertensão arterial, diabetes, absenteísmo, desmotivação, queda da produtividade, crises de ansiedade, insônia, variações de humor, relações conflituosas com os colegas e familiares, além de possíveis injúrias aos pacientes (RIBEIRO RP, et al., 2018).

A pandemia da COVID 19 é um agravante para o desenvolvimento do estresse laboral em profissionais da medicina e da enfermagem que estão na linha de frente, visto que a alta exposição a um vírus causador de uma doença desconhecida, muitas vezes sem equipamentos de proteção adequados, aumentou a pressão e o medo da contaminação individual e dos familiares (TEIXEIRA GS, et al., 2019). Por essa razão, muitos optaram pelo isolamento profilático e saíram de casa para garantir proteção aos entes queridos, no entanto o estado psíquico deles passou a flutuar entre a tranquilidade, a tristeza e a ansiedade em momentos de solidão (HORTA RL, et al., 2021).

Portanto, o presente estudo teve como objetivo a realização de um levantamento teórico acerca dos principais estressores responsáveis pelo desenvolvimento do estresse ocupacional agudo e crônico nos profissionais da medicina e da enfermagem, bem como analisar e descrever o impacto da COVID 19 na saúde mental. 


\section{REVISÃO BIBLIOGRÁFICA}

\section{Teorias sobre o estresse}

Com o advento da Revolução Industrial e do capitalismo, as relações entre patrões e empregados foram readaptadas de modo que o valor do trabalho se tornou diretamente proporcional à força física do indivíduo. Essa dinâmica era alicerçada nas jornadas exaustivas, nos baixos salários, no alto nível de estresse devido a tensão nas fábricas, bem como na consequente precariedade das condições de vida impactando negativamente o físico e o psicológico dos trabalhadores (SILVA RM, et al., 2018). Assim sendo, iniciaramse as discussões sobre a saúde e a segurança ocupacional, o que resultou na aprovação, em 1802, da Lei de Saúde e Moral dos Aprendizes, a primeira lei de proteção aos trabalhadores (MENDONÇA GS, et al., 2018).

Entre os anos de 1920 e 1930, o médico Hans Seyle apresentou ao mundo o conceito do estresse biológico, dividido em 3 fases. Primariamente, na fase de Alerta, o organismo na tentativa de manter a sua homeostase e se defender responde com taquicardia, sudorese e cefaleia por exemplo. Em seguida, a Resistência e sua adaptação aos estressores manifestada com o medo e a ansiedade, posteriormente instalase Exaustão, fase caracterizada pelo estresse crônico em que é perceptível a presença de doenças como a depressão (SILVA GN, 2018).

Lazarus, Lanier e Folkman correlacionaram o estresse biológico ao psicológico através da Teoria Interacionista, enfatizando que comportamentos e emoções teriam papéis fundamentais no desenvolvimento do estresse. Sob essa égide, a interação do homem com o meio ambiente e o modo como ele responde aos estressores será a chave para a presença ou não de prejuízos ao seu bem estar (SILVA RM, et al., 2018).

A psicóloga Marilda Lipp nos anos 2000 ratificou esses estudos e fomentou à importância do corpo e da mente no enfrentamento do transtorno do estresse em seu modelo quadrifásico: Alerta, Resistência, Quase Exaustão e Exaustão. A mudança apresentada inclui a fase da Quase Exaustão, período em que o indivíduo, apesar do cansaço físico e emocional ainda é capaz de manter a sua rotina, entretanto na Exaustão essa capacidade é perdida e a instalação das patologias é iminente (BANDEIRA MAD, 2017).

\section{Definição do estresse}

O estresse é uma resposta normal do organismo humano às diversas situações em que a adaptação é necessária, entretanto pode ser transformado em patológico, caso seja persistente e causador direto de injúrias a qualidade de vida (CAPRISTE MLP, et al., 2017). O tipo de resposta e a intensidade vai depender do agente estressor, de que maneira o indivíduo vai percebê-lo (HIRSCHLE ALT, et al., 2019) e do ambiente externo ao qual está inserido. Logo, as causas do transtorno do estresse podem ser endógenas e exógenas, respectivamente. Nesse contexto, se a percepção for positiva, a busca será por evolução com o enfrentamento das dificuldades impostas. Por outro lado, se negativa, inúmeras disfunções podem surgir, desde de transtornos mentais até distúrbios de natureza física (TABOSA MPO e CORDEIRO AT, 2018).

O estresse é uma síndrome de adaptação com uma clínica semelhante para a fase aguda e crônica, isso acontece porque o Sistema Nervoso Central não distingue ameaças, mas a duração dos sinais e sintomas desencadeados, pois o comando é o mesmo, lutar ou fugir (CAPRISTE MLP, et al., 2017). De acordo com a American Psychistric Association (APA) (2014), a resposta aguda acontece logo após a exposição ao estressor persistindo em até 72 horas, o indivíduo pode apresentar irritabilidade, tristeza, ansiedade e desespero. O crônico ou pós-traumático tem duração maior do que 4 semanas, resultando em uma clínica mais intensa em que a ansiedade pode evoluir para o transtorno do pânico e a tristeza para a depressão, além de outros distúrbios orgânicos.

\section{Estresse ocupacional}

Define-se estresse ocupacional como um desequilíbrio entre a capacidade de gerenciamento e/ou adaptação às demandas impostas pela instituição empregadora (SILVA LC e SALLES TLA, 2016). Nesse contexto, profissionais de todas as áreas podem ser acometidos, em virtude de diferentes fatores que o expõe ao desequilíbrio entre o trabalho e descontrole psíquico (FELIX D, et al., 2017). Os agentes estressores destacam-se como potenciais determinantes para a ocorrência dos transtornos mentais comuns (TMC), os 
quais correspondem a fadiga, insônia, irritabilidade, dificuldade de concentração e esquecimento, constituindo-se em um problema de saúde pública que impacta diretamente na esfera social, pessoal, além do financeiro (CAMPOS FM, et al., 2020). Por outro lado, o transtorno pode predispor ao desenvolvimento da síndrome metabólica, hipertensão, diabetes, além do absenteísmo e a iminente queda na produtividade (RIBEIRO RP, et al., 2018).

À fim de entender o processo de adoecimento e os impactos negativos na qualidade do trabalho e na saúde do trabalhador, inúmeros estudos começaram a ser desenvolvidos (FELIX D, et al., 2017). Todo esse processo perpassa por diferentes abordagens e respostas ao estresse, porém existe um consenso entre as literaturas que dividem as reações ao estresse em grupos que incluem fatores não intrínsecos ao trabalho como carga horária, a responsabilidade e a pouca clareza nas funções, relacionamentos interpessoais, dificuldade em tomar decisões, além dos conflitos familiares devido a exigências laborais e a tentativa de conciliação com as demandas e relacionamentos familiares (SILVA LC e SALLES TLA, 2016).

Segundo uma pesquisa realizada pelo International Stress Management Association no Brasil (ISMA-BR), $70 \%$ dos profissionais brasileiros sofrem com algum nível de estresse ocupacional, sendo decorrente das condições de trabalho, relações conflituosas, baixos salários entre outras (FELIX D, et al., 2017). Além disso, de acordo com a Organização Internacional do Trabalho (OIT) e a Organização Pan Americana de Saúde (OPAS), no Brasil, $14 \%$ dos benefícios referentes ao auxílio doença estão relacionados a desordens mentais (TABOSA MPO e CORDEIRO AT, 2018).

Os trabalhadores da saúde podem sofrer de estresse relacionado ao trabalho como resultado de fatores organizacionais e um desequilíbrio das demandas, habilidades e suporte social no trabalho ou qualquer combinação destes. Em ambientes hospitalares, estudos indicaram que o trabalho intenso, a carga horária exaustiva e a falta de clareza de papéis estão associados à ansiedade e a depressão entre médicos e enfermeiros (RUOTSALAINEN JH, et al., 2014).

\section{Medicina}

A profissão médica ainda figura na mente humana como um sacerdócio abarcando expectativas e pressões irreais, assim sendo a medicina continua romantizada como uma carreira de sucesso, totalmente imune aos problemas e adoecimentos (CONCEIÇÃO LS, et al., 2019). Essa visão deturpada esconde um ritmo de vida desgastante, iniciado ainda na graduação, sendo este o principal responsável pelo desequilíbrio entre o físico e o mental, tornando-se agente direto do surgimento de patologias de ordem psiquiátrica (BANDEIRA MAD, 2017).

Pesquisas relatam que os acadêmicos de medicina apresentam altos índices de sofrimento psíquico, esgotamento e ideação suicida em números maiores do que a população em geral, no Brasil e no mundo. Isso é explicado pela sobrecarga de conteúdo e a consequente falta de tempo para um aprendizado de qualidade que está associado à constante sensação de culpa e frustração por não conseguir atingir as próprias expectativas no curso e a pressão social a qual estão expostos (CONCEIÇÃO LS, et al., 2019). Desse modo, os estudantes iniciam um processo de adoecimento gradual caracterizado por lapsos de memória, irritabilidade, insônia, emagrecimento, ansiedade e outros sintomas que fomentam a insegurança, o medo de errar e a impotência diante das situações (DAMASO JGB, et al., 2019).

A construção do profissional médico perpassa por uma cultura tóxica em que práticas falíveis não são admitidas, por esse turno é importante salientar que o sofrimento experimentado desde de a graduação fortalece a não percepção do próprio estado de adoecimento, o seu prolongamento e a sua vulnerabilidade a situações de risco, legado invisível pertencente a classe médica (CONCEIÇÃO LS, et al., 2019).

Mesmo com as utopias, o profissional médico segue cumprindo o seu papel e pagando um preço alto demais. Estudos comprovam que entre os principais fatores de risco para o acometimento mental e físico estão o descontentamento com o sistema de saúde, a baixa remuneração diante da imensa responsabilidade, os múltiplos empregos, além dos distúrbios do sono, irritabilidade, o abuso de substâncias como álcool e drogas como uma tentativa desesperada para amenizar o cansaço e o sofrimento (GRACINO ME, et al., 2016). Os níveis de estresse ocupacional variam de acordo com as especialidades, destacando-se os médicos da Unidade de Terapia Intensiva (UTI) e os cirurgiões (BANDEIRA MAD, 2017). 
É importante salientar que os residentes, principalmente os do primeiro ano, podem ter esse estresse potencializado, visto vez que além de médicos são estudantes. Isso implica em ter que manter o equilíbrio para lidar com a avaliação e o julgamento dos preceptores, alunos e dos residentes dos outros anos, os " $R$ mais", aliado aos afazeres acadêmicos paralelos e a uma rotina peculiar nos hospitais, que muitas vezes pode gerar tristeza e frustração (KATSURAYAMA M, et al., 2011).

Diante do exposto, pode-se afirmar que o médico é o profissional da saúde que mais trabalha, no quesito carga horária, o que é um fator preponderante para o desenvolvimento de patologias psíquicas. Dados de um estudo demonstram que $29 \%$ dos médicos apresentam sintomas elevados para a depressão, $24 \%$ para ansiedade e $15 \%$ para o transtorno do estresse pós-traumático. Sendo assim, o uso de substâncias por médicos da emergência, bem como o desenvolvimento de doenças cardiovasculares por cirurgiões são mais frequentes do que em outras especialidades (BERNBURG M, et al., 2016).

Essa realidade é nomeada como mal estar, tristeza, adoecimento mental e físico que impacta diretamente na qualidade da assistência aos pacientes e nas relações interpessoais com os colegas, tornando-as mais superficiais. Sob essa égide, os médicos apresentam uma maior taxa de tentativas de suicídio, divórcio e ao abuso de drogas, se comparado a maioria da população (DIAS EC, 2015).

\section{Enfermagem}

Florence Nightingale é considerada a mãe da enfermagem moderna e a grande responsável por abarcar o acolhimento humano e o gerenciamento do cuidado no âmbito administrativo. A mesma, responde pela construção de um legado que ainda hoje reverbera, no que tange sobre a importância dos cuidados da enfermagem, o estabelecimento real da função do enfermeiro, bem como a construção e a valorização da classe no futuro (CARRARO TE, 2013). Ainda hoje, não mais Florence, mas o Conselho Regional de Enfermagem (COREN) continua lutando por reconhecimento profissional, pela aprovação da jornada de trabalho de 30 horas, bem como o aumento do piso salarial - o principal estressor da classe é a jornada longa em vários serviços para suprir o financeiro (SOUSA CNS, et al., 2020).

A principal força de trabalho das instituições hospitalares é o corpo de enfermagem que abrange técnicos e enfermeiros, os quais respondem pelo cuidado diário, 24 horas por dia e 7 dias por semana. A assistência direta é um grande desafio, pois perpassa não somente pelo acolhimento, curativos, administração de medicamentos, mas principalmente pelo gerenciamento de pessoas no serviço para que tudo funcione de forma concreta (CARVALHO DP, et al., 2017).

Técnicos e enfermeiros lidam diuturnamente com a falta de insumos físicos (curativos, gaze, medicamentos entre outros) e humanos os quais são fatores primordiais para o desenvolvimento do estresse agudo ou crônico que corrobora com a ansiedade, insônia, alienação, angústias (SOUSA CNS, et al., 2020), além do absenteísmo, piora da qualidade de vida, da insatisfação laboral o que contribui para o aumento da doenças cardiovasculares, uma vez que a demanda de trabalho é maior que o suporte (UENO LGS, et al., 2017). Os profissionais atuantes na Terapia Intensiva (UTI) e no Centro cirúrgico estão entre os que mais sofrem com o transtorno do estresse (SOUSA CNS, et al., 2020).

A complexidade da UTI já é considerada um agente estressor, pois os profissionais lidam diretamente com a vida e com a morte, além da relação de proximidade com os familiares. Além disso, o barulho das máquinas, as condições inadequadas, as equipes pouco entrosadas e a redução de funcionários frente a complexidade dos procedimentos fortalecem o aumento das doenças e agravos (SANTOS MJ e GUEDES VM, 2019).

No centro cirúrgico (CC) a assistência eletiva e/ou de emergência estressa a enfermagem devido a rotatividade de procedimentos e a iminente permanência por mais tempo no bloco cirúrgico, logo conflitos interpessoais tornam-se mais fáceis de acontecer. Aliado a isso, a carga emocional e a esperança que os familiares depositam no corpo clínico é um estressor agravante, ainda que inconsciente, porque nem sempre o paciente se recupera. Por esse turno, o organismo responde ao estresse com dor lombar, cefaleia, gastrite e transtornos de ansiedade, além de outros problemas que começam a fazer parte da rotina de técnicos e enfermeiros (SORATTO MT, et al., 2016). 


\section{O impacto do COVID}

Em dezembro de 2019 o primeiro alerta de uma pneumonia de causa desconhecida foi anunciado, na qual várias autoridades de saúde locais de Wuhan, província de Hubei, na China, associaram epidemiologicamente a causalidade e epicentro, ao mercado de frutos do mar em Wuhan (ZHU N, et al., 2020). Em janeiro de 2020, a Organização Mundial da Saúde (OMS) declarou o surto do novo coronavírus (COVID-19), apontando como uma Emergência de Saúde Pública de Preocupação Internacional (ESPII). Em março de 2020, a OMS avaliou que a COVID-19 seria caracterizada como uma pandemia. Indubitavelmente, proteger o físico e o emocional dos profissionais da linha de frente foi identificado como algo de suma relevância para a garantia da assistência aos pacientes acometidos pelo novo coronavírus (WORLD HEALTH ORGANIZATION 2020).

Lidar com o desconhecido é um desafio para qualquer pessoa, no entanto para aqueles que têm a vida do outro como responsabilidade é algo muito maior. Manter o equilíbrio mental tornou-se um desafio, uma vez que a prevenção e o tratamento para um vírus altamente patogênico e que estava matando milhares de pessoas era desconhecido (HORTA RL, et al.,2021). O mundo já enfrentou inúmeros desastres biológicos como o EBOLA, a Influenza H1N1 e a Síndrome Respiratória Aguda (SARS), o denominador comum entre eles foi $O$ isolamento social como medida prioritária para tentar frear a disseminação dos patógenos. Entretanto, a quarentena foi e é a grande responsável pelo impacto negativo na saúde mental de profissionais médicos e enfermeiros durante e após os períodos epidêmicos (CABARKAPA S, et al.,2020).

Trabalhar na linha de frente é estar exposto a uma alta carga viral, ao risco iminente de contaminação, a condições de trabalho por vezes inadequadas, sem equipamentos de proteção individual (EPIs), associado ao déficit humano, jornadas de trabalho exaustivas, alicerçada no estresse individual e coletivo que é desencadeador de reações físicas e emocionais (HORTA RL, et al., 2021). Assim sendo, destacam-se o sedentarismo, o aumento de doenças cardiovasculares, diabetes mellitus, bem como queixas relacionadas a insônia, associada ao uso de medicamentos indutores do sono, logo é importante salientar que entre os profissionais brasileiros ainda houve um aumento de $27 \%$ no consumo de álcool (MOTA IA, et al., 2021).

Pesquisas feitas com médicos em Wuhan relatam o fortalecimento dos sintomas que convergem para a ansiedade, depressão, para o abuso de drogas, além de prejuízo à qualidade do sono, bem como o medo obsessivo quanto à infecção individual e dos familiares. Em relação aos enfermeiros destaca-se a depressão, a ansiedade, a insônia e a angústia, principalmente entre as mulheres. Uma vez acometidos por esses transtornos, a tendência dos profissionais é a deficiência na assistência movida pelo déficit de atenção (TEXEIRA CFS, et al., 2020).

É importante ratificar que as respostas aos agentes estressores podem ser diferentes, nem todos os profissionais reagem da mesma forma, isso significa que alguns evoluem positivamente, entretanto outros mantém os transtornos por tempo mais prolongado (WALTON M, et al., 2020). O transtorno da ansiedade, a depressão e o estresse pós-traumático estão entre as doenças de maior impacto negativo da pandemia da COVID 19 na saúde mental dos profissionais médicos e enfermeiros da linha de frente (TALEVI D, et al., 2020).

\section{CONIDERAÇÕES FINAIS}

Diante do exposto, os estressores generalizados irão surgir ou se agravar, logo cabe a reflexão a respeito da inexistência de programas institucionais que priorizem o cuidado para com a saúde mental dos profissionais da medicina e da enfermagem. Ambas as classes são reféns do estresse ocupacional há décadas e impedidos, ainda que de forma velada, de manifestar suas vulnerabilidades em virtude do grau de responsabilidade e funções diante da sociedade. Desse modo, o estresse se tornou uma constante e o agente principal das crises de ansiedade que impactam o físico e o psicológico, sendo agravadas ou manifestas, inclusive, em momentos de pós pandemias. Assim, é imprescindível uma mudança de postura dos serviços de saúde, a fim criar estratégias para o início e a manutenção dos cuidados físico e mental dos profissionais da medicina e da enfermagem. 


\section{REFERÊNCIAS}

1. AMERICAN PSYCHISTRIC ASSOCIATION. Manual diagnóstico e estatístico de transtornos mentais (DSM-5). Porto Alegre: Artmed, 2014; 284p.

2. BANDEIRA MAD. Estresse ocupacional em médicos: uma revisão bibliográfica. Revista Amazônia Science \& Health, 2017; 5(2): 25-32.

3. BERNBURG M, et al. Physicians' occupational stress, depressive symptoms and work ability in relation to their working environment: a cross-sectional study of differences among medical residents with various specialties working in German hospitals. British Medical Journal, 2016; 6: e-011369.

4. CABARKAPA S, et al. The psychological impact of COVID-19 and other viral epidemics on frontline healthcare workers and ways to address it: A rapid systematic review. Brain, behavior, \& immunity - health, 2020; 8: 100144.

5. CAMPOS FM, et al. Estresse ocupacional e saúde mental no trabalho em saúde: desigualdades de gênero e raça. Cadernos de Saúde Coletiva, 2020; 28(4):579-589.

6. CAPRISTE MLP, et al. Reflexões sobre a influência do estresse crônico na transformação de células saudáveis em células cancerígenas. Revista de Enfermagem UFPE online, 2017; 11(6): 2473-2479.

7. CARVALHO DP, et al. Cargas de trabalho e a saúde do trabalhador de enfermagem: revisão integrativa Revista Cogitare Enfermagem, 2017; 22 (1): 01-11.

8. CONCEIÇÃO LS, et al. Saúde mental dos estudantes de medicina brasileiros: uma revisão sistemática da literatura. Revista da Avaliação da Educação Superior, 2019; 24 (3): 785-802.

9. DAMASO JGB, et al. É muita pressão! Percepções sobre o desgaste mental entre estudantes de medicina. Revista Brasileira de Orientação Profissional, 2019; 20 (2): 29-41.

10. DIAS EC. Condições de trabalho e saúde dos médicos: uma questão negligenciada e um desafio para a Associação Nacional de Medicina do Trabalho. Revista Brasileira de Medicina do Trabalho, 2015; 13(2):60-68.

11. FELIX DB, et al. Análise dos níveis de estresse no ambiente hospitalar: um estudo com profissionais da área de enfermagem. Revista de Carreiras e Pessoas, 2017; 7 (2): 530-543.

12. FRELLO AT, CARRARO TE. Contribuições de Florence Nightingale: uma revisão integrativa da literatura. Escola Anna Nery Revista de enfermagem, 2013; 17(3): 573-579.

13. GRACINO ME, et al. A saúde física e mental do profissional médico: uma revisão sistemática. Revista Saúde em Debate, 2016; 40(110): 244-263.

14. HIRSCHLE ALT, et al. Estresse e bem-estar no trabalho: o papel moderador da regulação emocional. Revista Psicologia Organizações e Trabalho, 2019; 19(1): 532-540.

15. HONORATO CMA, MACHADO FCA. Fatores desencadeantes do estresse laboral na emergência médica: uma revisão integrativa. Revista Ciência Plural, 2019; 5(1): 52-70.

16. HORTA RL, et al. O estresse e a saúde mental de profissionais da linha de frente da Covid-19 em hospital geral. Jornal Brasileiro de Psiquiatria, 2021; 70(1): 30-38.

17. KATSURAYAMA M, et al. Avaliação dos níveis de estresse psicológico em médicos residentes e não residentes de hospitais universitários. Revista da Sociedade Brasileira de Psicologia Hospitalar, 2011; 9(1): 75-96.

18. MENDONÇA GS, et al. Evolução histórica da saúde ocupacional. Revista Científica Multidisciplinar da UniSãoJosé, 2018; 11(1): 03-15.

19. MOTA IA, et al. Impact of COVID-19 on eating habits, physical activity and sleep in Brazilian healthcare professionals. Revista Arquivos de Neuropsiquiatria, 2021; 79(5): 429-436.

20. PRADO CEP. Estresse ocupacional: causas e consequências. Revista Brasileira de Medicina do Trabalho, 2016; 14(3): 285-289.

21. RIBEIRO RP, et al. Estresse ocupacional entre trabalhadores de saúde de um hospital universitário. Revista Gaúcha de Enfermagem, 2018; 39: e-65127.

22. RUOTSALAINEN JH, et al. Preventing occupational stress in healthcare workers. Revista Paulista de Medicina, 2014; 134(1): 92.

23. SADIR MA, et al. Stress e qualidade de vida: influência de algumas variáveis pessoais. Paideia (Ribeirão Preto), 2010; 20(45): 73-81.

24. SANTOS MJ, GUEDES VM. Estresse entre profissionais de enfermagem em unidade de terapia intensiva. Revista Científica de Enfermagem, 2019; 9(27):13-22.

25. SILVA GN. (Re)conhecendo o estresse no trabalho: uma visão crítica. Revista Interinstitucional de Psicologia, 2018; 12(1): 51-61.

26. SILVA LC, SALLES TLA. O estresse ocupacional e as formas alternativas de tratamento. Revista de Cerreiras e Pessoas, 2016; 6(2): 234-247.

27. SILVA RM, et al. Evolução histórica do conceito de estresse. Revista Científica Sena Aires, 2018; 7(2): $148-156$.

28. SORATTO MT, et al. O estresse da equipe de enfermagem no centro cirúrgico. Revista Interdisciplinar de Estudos em Saúde, 2016; 5(1): 179-192.

29. SOUSA CNS, et al. Análise do estresse ocupacional na enfermagem: revisão integrativa. Revista Eletrônica Acervo Saúde, 2020; (52): e-3511. 
30. TABOSA MPO, CORDEIRO, AT. Estresse ocupacional: análise do ambiente laboral de uma cooperativa de médicos de Pernambuco. Revista de Carreiras e Pessoas, 2018; 8(2): 282-303.

31. TALEVI D, et al. Mental health outcomes of the Covid-19 pandemic. Rivista di psichiatria, 2020; 55(3): $137-144$.

32. TEIXEIRA CFS, et al. A saúde dos profissionais de saúde no enfrentamento da pandemia de Covid-19. Revista Ciência \& Saúde Coletiva, 2020; 25(9): 3465-3474.

33. TEIXEIRA GS, et al. Qualidade de vida no trabalho e estresse ocupacional da enfermagem em unidade de pronto atendimento. Revista Texto \& Contexto Enfermagem, 2019; 28: e20180298.

34. UENO LGS, et al. Estresse ocupacional: estressores referidos pela equipe de enfermagem. Revista de Enfermagem UFPE online, 2017; 11(4): 1632-1638.

35. ZHU N, et al. A novel coronavirus from patients with pneumonia in China. The New England Journal of Medicine, 2020; 382:727-733.

36. WALTON M, et al. Mental health care for medical staff and affiliated healthcare workers during the COVID-19 pandemic. European heart journal, 2020; 9(3): 241-247.

37. WORLD HEALTH ORGANIZATION (WHO). Mental Health and Psychosocial Considerations during the COVID-19 Outbreak. Disponível em: https://www.who.int/docs/default-source/coronaviruse/mental-health-considerations.pdf. Acesso em: 21 de Maio de 2021. 